\title{
An improved ATP FRET sensor for yeast shows heterogeneity during nutrient transitions
}

\author{
Dennis Botman ${ }^{1}$, Johan H. van Heerden ${ }^{1}$ \& Bas Teusink ${ }^{1 *}$ \\ ${ }^{1}$ Systems Biology Lab/AIMMS, Vrije Universiteit Amsterdam, De Boelelaan 1085, 1081 HV, Amsterdam, \\ The Netherlands. \\ *Correspondence should be addressed to Bas Teusink (email: b.teusink@vu.nl)
}

Movie S1. Ratiometric movie of W303-1A WT cells expressing yAT1.03. Cells were grown on 1\% EtOH and pulsed with 100 $\mathrm{mM}$ glucose. Colour indicates the FRET ratio, shown by the calibration bar in the upper left.

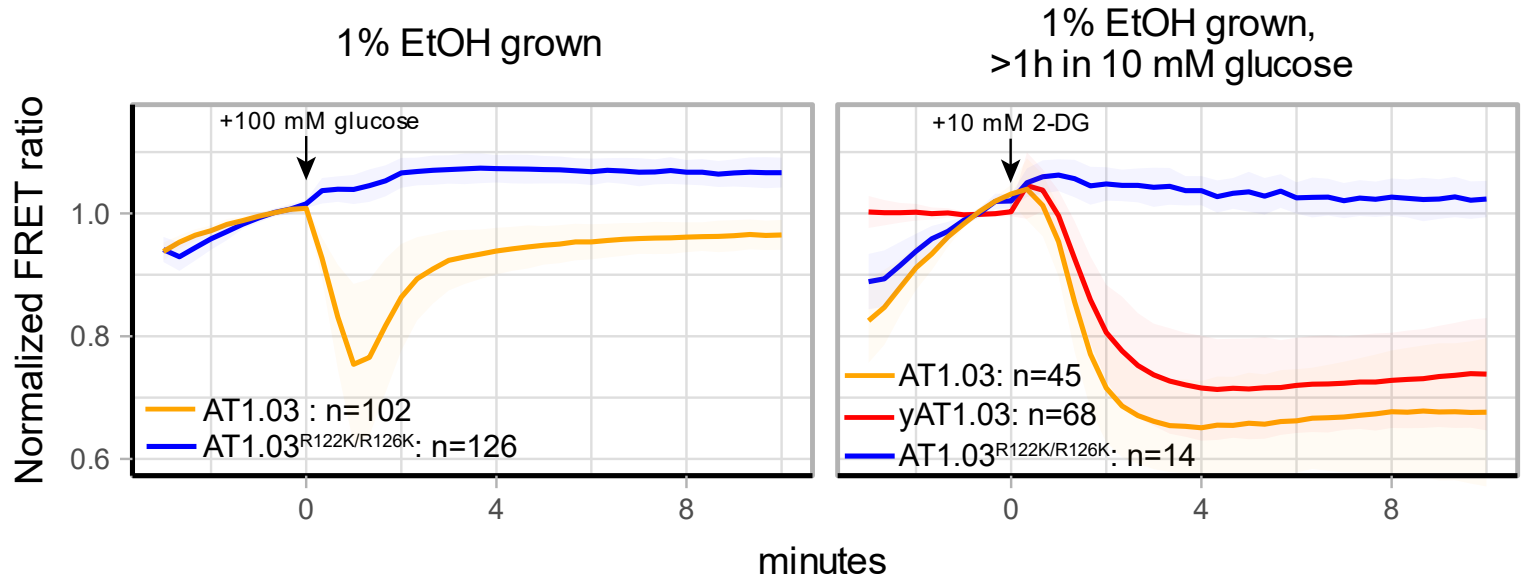

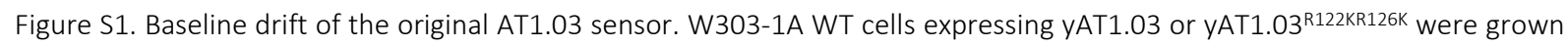
with $1 \% \mathrm{EtOH}$ as carbon source. Before the 2-deoxyglucose pulse, cells were incubated in $10 \mathrm{mM}$ glucose for at least 1 hour. As reference, the yAT1.03 sensor was plotted as well for this condition. At $t=0$ minutes, glucose was added and the FRET responses were measured. Lines show mean responses, normalized to the 5 last frames before glucose addition, shades indicate SD.

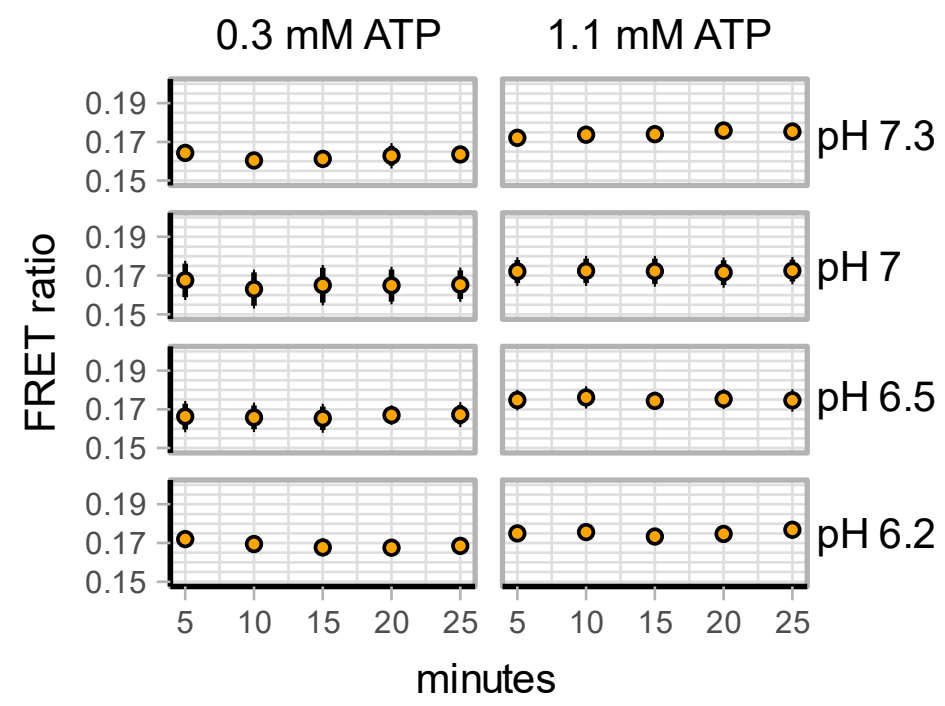


Figure S2. ATP levels in the cell-free extracts remain constant. The change in FRET level at either 0.3 or $1.1 \mathrm{mM}$ ATP was recorded for up to 25 minutes at various pH values. Points show mean FRET ratio of 3 replicates, errorbars indicate standard deviation.
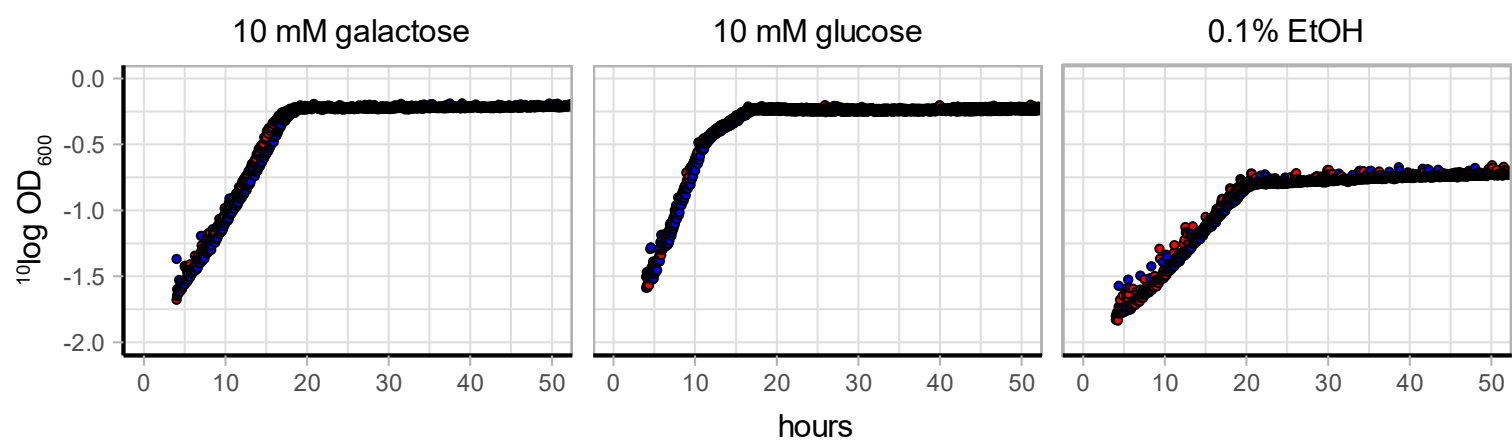

- yAT1.03 - pDRF1-GW

Figure S3. Growth of W303-1A cells expressing either yAT1.03 or the empty vector pDRF1-GW. Cells were grown to midlog with $0.1 \% \mathrm{EtOH}$ as substrate. Next, cells were washed and incubated for 10 minutes in $1 \mathrm{x}$ YNB containing no carbon source. Next, cells were transferred to $1 x$ YNB containing either $10 \mathrm{mM}$ galactose, $10 \mathrm{mM}$ glucose or $0.1 \% \mathrm{EtOH}$ and $\mathrm{OD}_{600}$ was measured. Dots depict the ${ }^{10} \log$ value of the $O D$, colours indicate the strain.

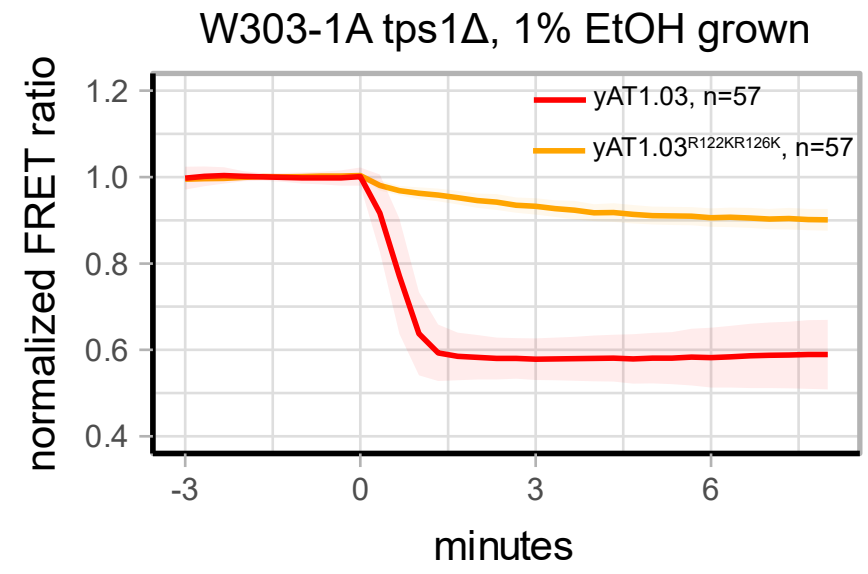

Figure S4. Response of W303-1A tps1 $\triangle$ cells expressing YAT1.03 and YAT1.03 ${ }^{\text {R122KR126K }}$ under severe perturbations. Cells were grown to midlog with $1 \% \mathrm{EtOH}$ as a carbon source and pulsed with $5 \mathrm{mM}$ of glucose at $\mathrm{t}=0$ minutes to induce a glycolytic imbalanced state. Lines show mean responses, normalized to the baseline (before perturbation), shades indicate SD. 


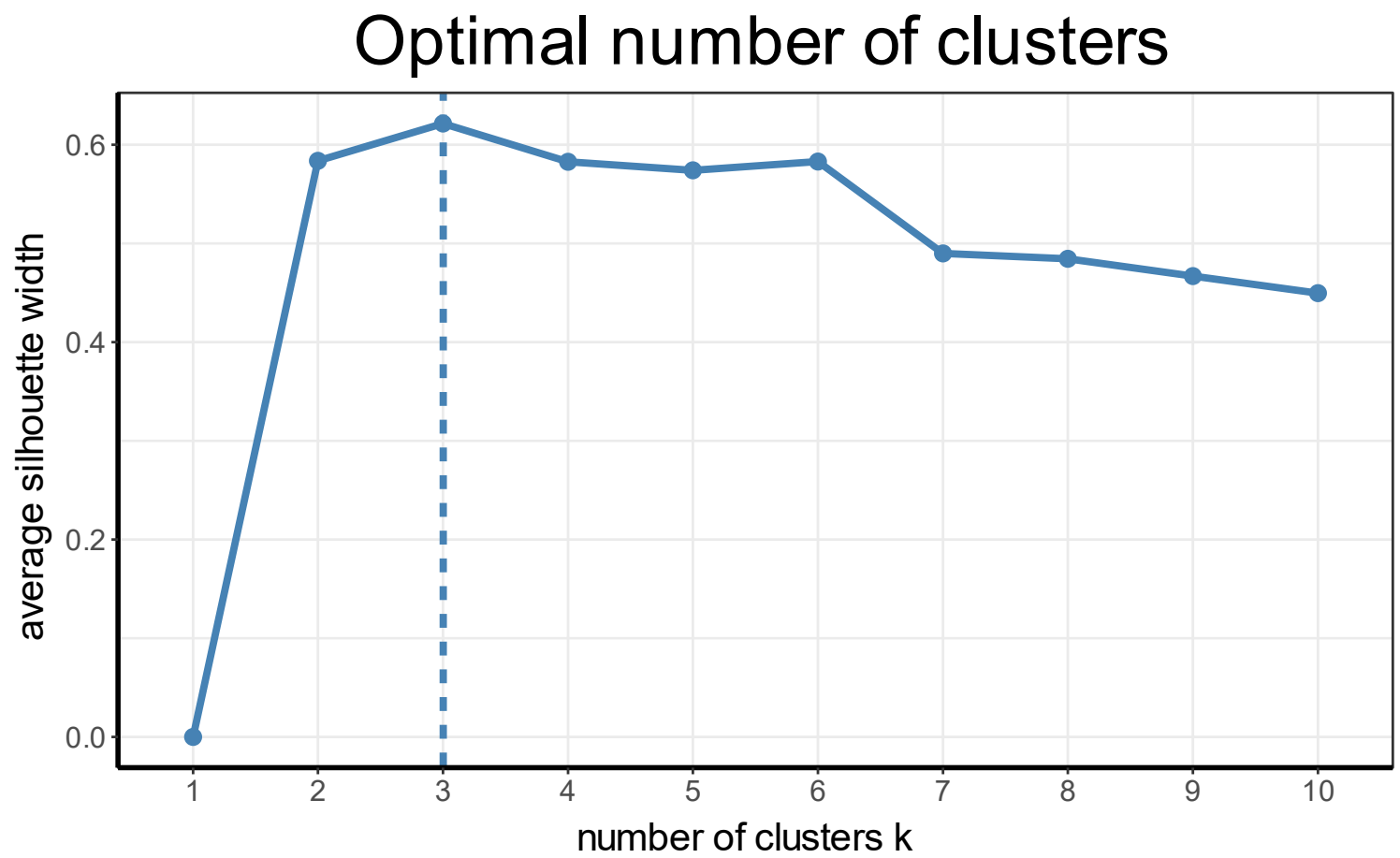

Figure S5. Determination of the optimal number of clusters. Three clusters were chosen based on the silhouette method, using the fviz_nbclust function in R.
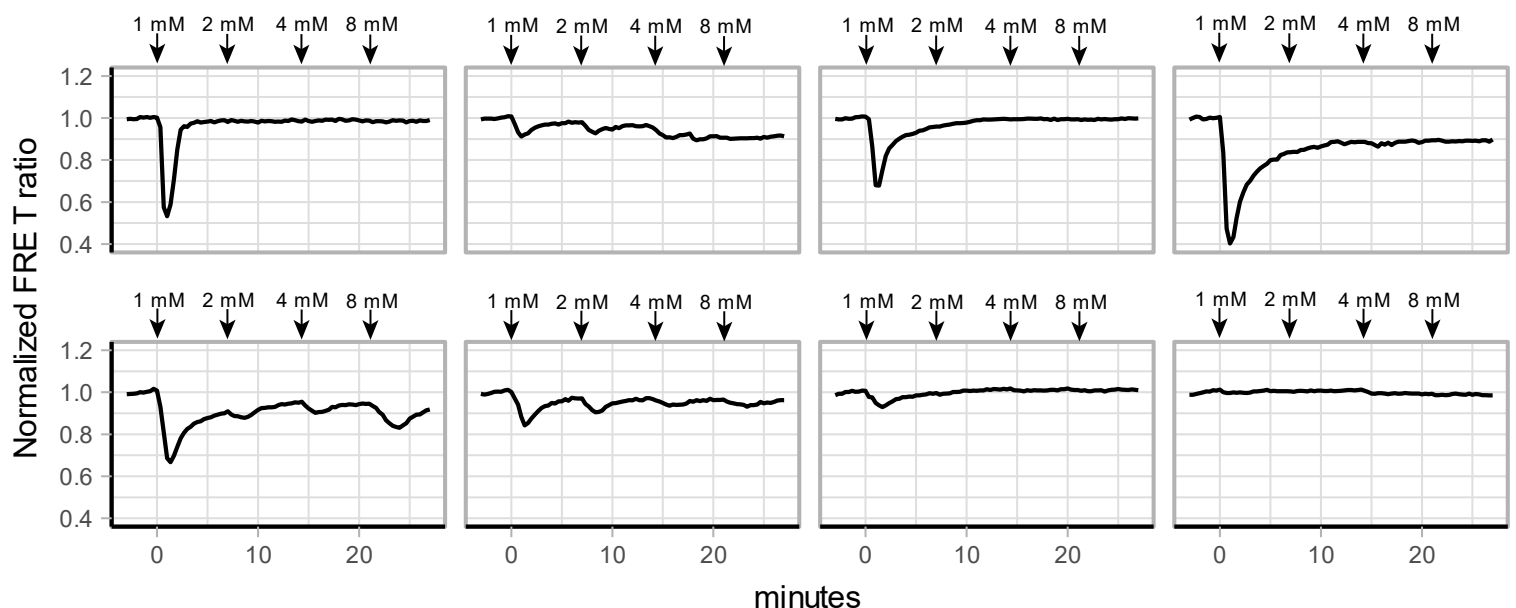

Figure S6. Single-cell FRET trajectories of cells exposed to multiple glucose pulses. W303-1A cells expressing yA1.03 were grown on $1 \% \mathrm{EtOH}$ and pulsed multiple times with increasing amounts of glucose. Every graph depicts the ATP response of a singlecell. Arrows indicate point of the new glucose concentrations reached. Lines show single-cell traces, normalized to the baseline. 


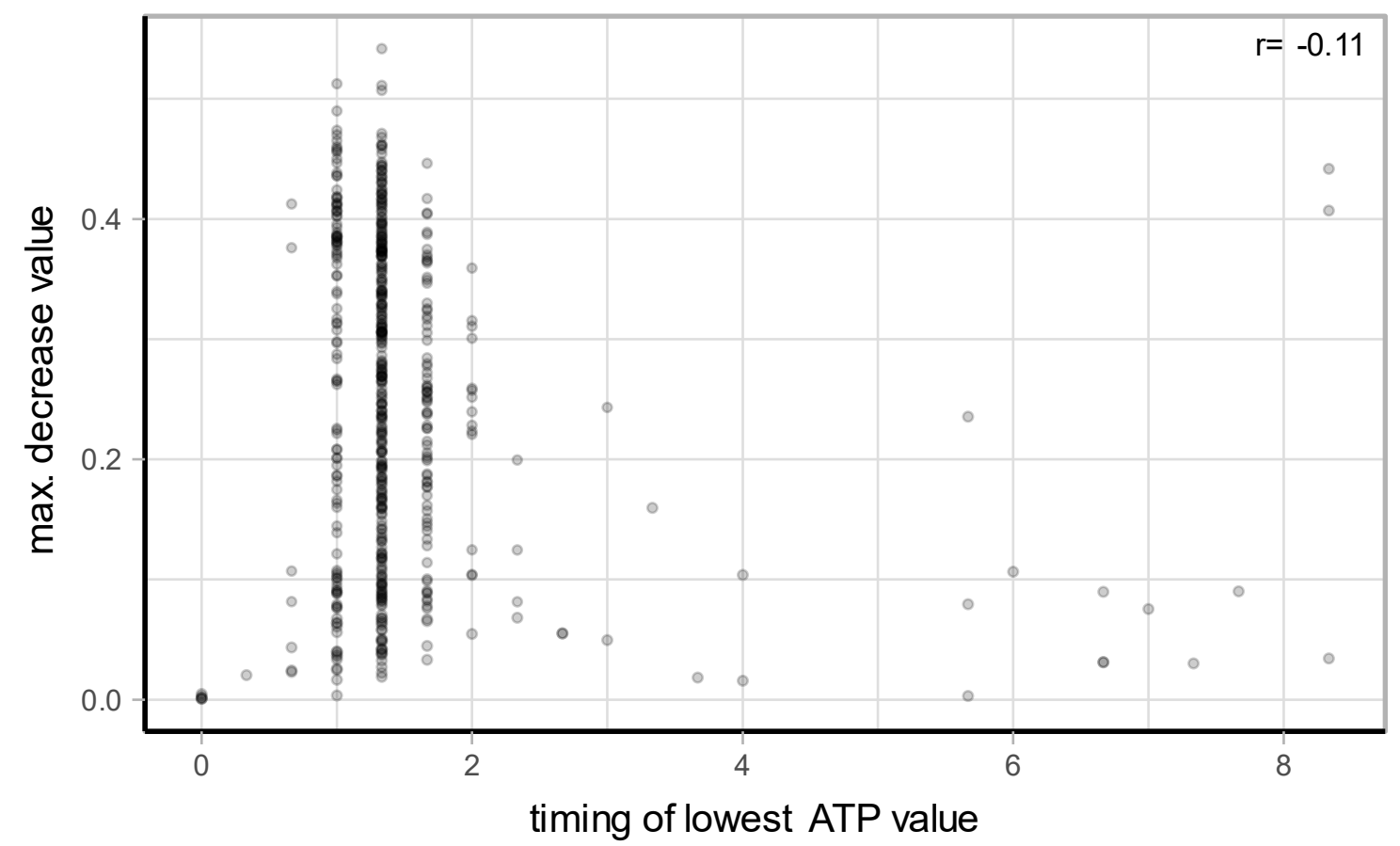

Figure S7. Correlation between the maximum decrease of ATP in a cell and the timing of this observed dip. Points show singlecell values. The spearman correlation coefficient ( $r$ ).

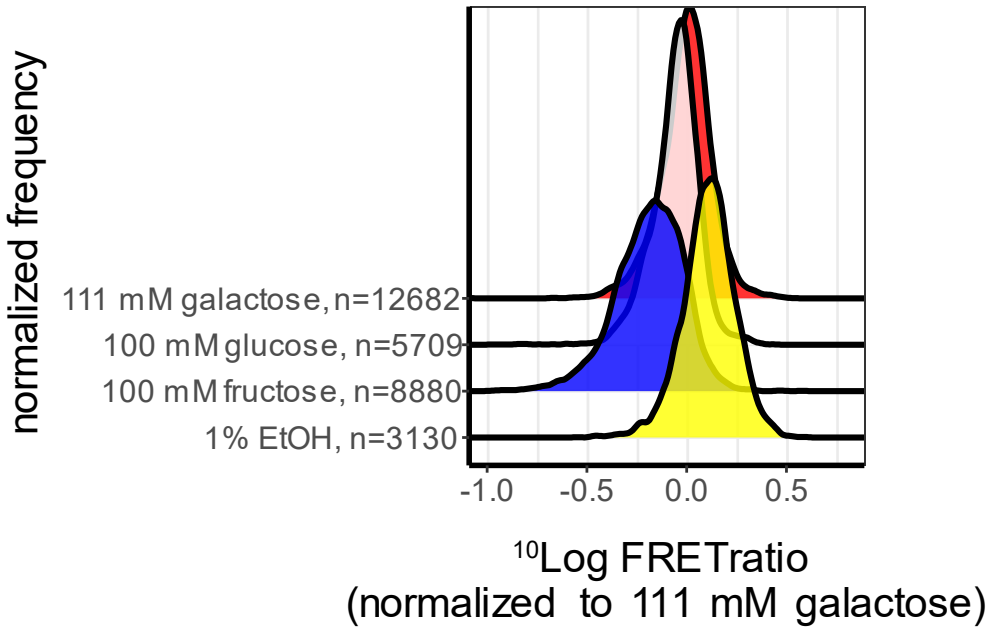

Figure S8. Steady-state ATP levels of W303-1A cells grown on various substrates. Normalized frequency distributions of W303$1 \mathrm{~A}$ cells grown on $1 \% \mathrm{EtOH}, 100 \mathrm{mM}$ fructose, $100 \mathrm{mM}$ glucose or $111 \mathrm{mM}$ galactose. FRET values were normalized to cells grown on $111 \mathrm{mM}$ galactose. 


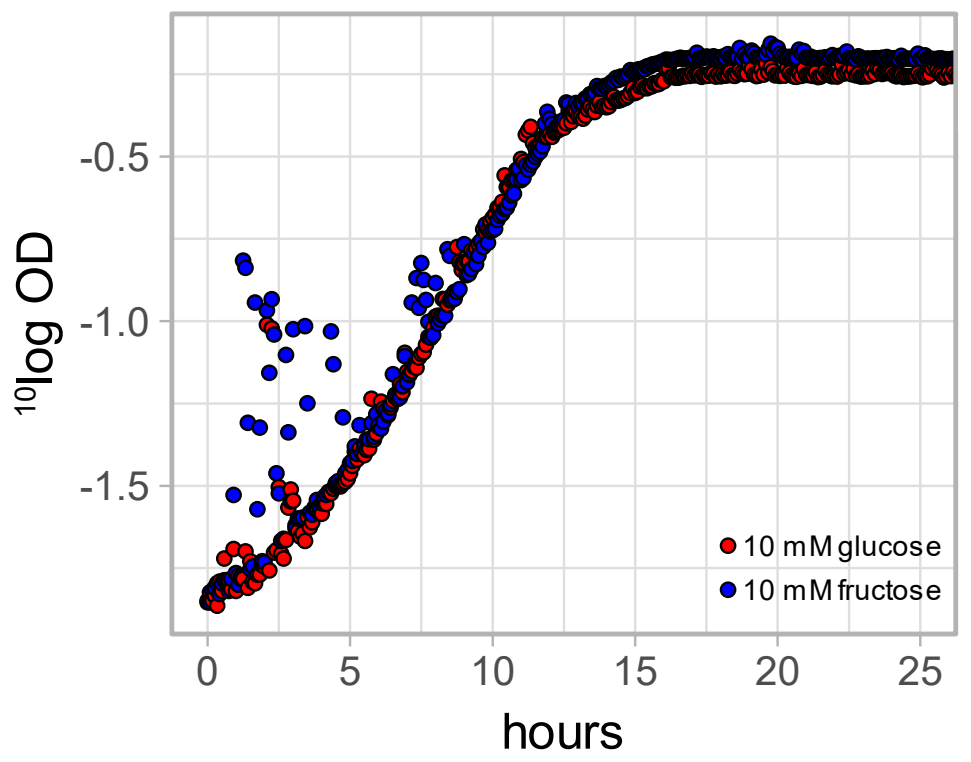

Figure S9. Growth of W303-1A on $10 \mathrm{mM}$ glucose or fructose. Cells were grown to midlog with $0.1 \%$ EtOH as substrate, washed and incubated for 10 minutes in $1 \times$ YNB containing no carbon source. Cells were subsequently transferred to $1 \times$ YNB containing either $10 \mathrm{mM}$ glucose or $10 \mathrm{mM}$ fructose and $\mathrm{OD}_{600}$ was measured. Dots depict the ${ }^{10}$ log value of the $\mathrm{OD}$, colours indicate the strain. 